\title{
EDITORIAL (PRO) RE MINIMALLY INVASIVE PORT-ACCESS MITRAL VALVE SURGERY
}

\author{
Edward D. Verrier, MD
}

$T^{\mathrm{w}}$ wo fundamental issues are raised by publication of the article titled "Minimally Invasive PortAccess Mitral Valve Surgery," by Professor Mohr and associates from the University of Leipzig (see page 567). First, should The Journal of Thoracic and Cardiovascular Surgery and other surgical journals publish "learning curve/feasibility" results after the introduction of a new surgical technology? Second, how should medicine, or cardiothoracic surgery in particular, safely introduce new technology into the competitive marketplace at a time when evidence based, outcome measured results are increasingly demanded by insurers, government agencies, and patients?

In regard to the first question, I believe the Journal has a responsibility to publish not only scientifically valid basic science research and prospective randomized clinical trials, but also articles that might affect safety and efficacy issues, even if not optimally designed. If one looks at articles published in the cardiothoracic literature historically and more recently, the majority of articles remain retrospective reviews, personal series, case reports, or megaanalyses. Technology evolves so rapidly that by the time a prospective randomized trial might be designed and carried out, the variables have changed and the results are no longer applicable.

The presentation of Professor Mohr's experience with Port-Access mitral valve surgery at the 1997 meeting of The American Association for Thoracic Surgery, along with the publication of the abstract, may have already contributed to the dramatic improvements in results. Abstracts submitted for re-

From the Division of Cardiothoracic Surgery, University of Washington, Seattle, Wash.

Requested for publication Sept. 23, 1997; received Dec. 2, 1997; accepted for publication Dec. 5, 1997.

Address for reprints: Edward D. Verrier, MD, Division of Cardiothoracic Surgery, University of Washington, Box 356310, 1959 NE Pacific St., Seattle, WA 98195.

J Thorac Cardiovasc Surg 1998;115:565-6

Copyright (c) 1998 by Mosby, Inc.

$0022-5223 / 98 \$ 5.00+0 \quad \mathbf{1 2 / 6 / 8 8 0 1 0}$

cent cardiothoracic surgery and cardiology meetings have documented dramatically better results with the Port-Access technology and have identified a number of critical issues: (1) There is a learning curve, so training is essential; (2) femoral artery and groin wound complications do occur, and local vascular reconstruction after cannula removal must be meticulous; (3) the descending aorta presents potentially lethal complications if not investigated before the operation (by transesophageal echocardiography or computed tomographic scanning) and respected during the operation; (4) neuropsychologic complications are not decreased with endovascular aortic occlusion and probably occur at a higher incidence because of inadequate deairing, endovascular manipulation, retrograde aortic perfusion, and balloon manipulation in close proximity to the aortic arch; (5) endoaortic balloon migration is common, may be difficult to deal with during the operation, and is potentially dangerous; (6) mitral valve disease must be carefully assessed to avoid a high reoperative rate (6/51 patients in the present study); (7) respiratory management must be conducted aggressively in the postoperative period to avoid prolonged ventilatory management; (8) at present, this particular operative method should not be chosen on the basis of presumed reduction in cost.

Surgeons considering using the Port-Access system, or any unfamiliar surgical technique, for that matter, must be aware of all potential complications and have strategies developed in advance to avoid complications or to deal with them if they arise. A report such as Professor Mohr's assists surgeons in developing these strategies and therefore is a valuable part of the medical literature.

With regard to the second question, as a specialty we must think through the issues that apply to advances in surgical techniques. If we look at our specialty in historic terms, modern cardiac surgery is about fifty years old and thus is still in its infancy. Major changes have come rapidly with the introduction of cardiopulmonary bypass, myocardial revascularization, valve replacement and repair techniques, and more recently minimally invasive surgical approaches, transmyocardial laser revascu- 
larization, and ventricular reduction surgery. Such rapid and impressive advances may not have occurred if the medical society, insurance companies, and government agencies demanded that every innovation undergo the scrutiny of a prospective randomized trial. Coronary artery bypass grafting underwent such scrutiny (albeit expensive and labor intensive) with the Coronary Artery Surgery Study (CASS), ${ }^{1}$ funded by the National Institutes of Health, and the European Coronary Artery Surgery Study Group, ${ }^{2}$ and these studies continue to define today's standards for coronary artery bypass surgery. However, most of the techniques used in modern cardiac surgery were not subjected to randomized trials but nonetheless have been carefully scrutinized and are continually being refined. Indeed, the early advances in cardiac surgery occurred because innovative surgeons were willing to "push the envelope." Certainly the invasive cardiology community has little regulation as they introduce new catheterbased approaches on what seems like a monthly basis. The bottom line, however, is that our population is living longer than ever before, and much of that longevity has been due to advances in treating the number one killer-heart disease.

The other side of the coin reflects the recent pressure within the United States scientific community (National Institutes of Health), governmental regulatory agencies (Food and Drug Administration, Health Care Financing Administration), and purchasers of care (Medicare, Blue Cross/Blue Shield) demanding evidence-based outcomes analysis. ${ }^{3}$ Such analysis is viewed as critical before the release of certain new technologies and in many instances before authorizing payment. This process of regulation has gone on for a number of years but is accentuated by managed care priorities and the health care spending crisis. ${ }^{4}$ The more common cardiac surgery procedures have become standardized, providing a benchmark for comparison of newer procedures and technologies. These standards will be increasingly used by both the scientific community and purchasers of health care to judge the safety, efficacy, and utility of new developments.

In recent years there has been a subtle but real pressure to discourage clinical innovations in the United States for a variety of complex reasons. The end result, however, is that most of the clinical advances in cardiothoracic surgery have originated outside the United States (the Jatene operation for transposition, the Carpentier operations for mitral valve disease, the Fontan operation for single ventricle physiology, the David operation for aortic valve repair, the Batista operation for cardiomyopathy, and others). Even the present series, presented by Mohr and associates, originates from Europe. More recently, authorization for payment in the Medicare population has been withheld for transmyocardial laser revascularization and reduction ventriculoplasty pending more definitive outcomes analyses showing not only feasibility but also safety and efficacy. Despite widespread press in the lay literature and promotion by aggressive surgeons looking to increase market share, clinical innovations will increasingly be scrutinized before widespread acceptance or payment will occur. The continued development of minimally invasive cardiac surgery will depend on finding a middle ground between the forces that encourage innovation and advancement and those that repress it.

The debate over minimally invasive surgery will continue, and I suspect these techniques will be modified and improved and ultimately will find a clinical niche. All of us in cardiothoracic surgery should critically review these technologic advances with a thoughtful and responsible approach to maximize clinical results for our individual patients. Do not use these innovative techniques for the wrong reasons. Experience and patient selection will be critical to obtaining excellent results. Use all methods of communication, such as our thoracic journals, formal society-sponsored meetings, industry-sponsored workshops, the Internet, and personal communication to form a solid foundation before instituting such programs. Finally, we must be respectful of our surgical heritage and support clinical innovation that is done responsibly and for the right reasons.

\section{REFERENCES}

1. Coronary Artery Surgery Study (CASS). A randomized study of coronary artery bypass surgery. Circulation 1983;68:951-9.

2. European Coronary Artery Surgery Study Group. Long-term results of prospective randomized study of coronary artery bypass surgery in chronic stable angina pectoris. Lancet 1982; 2:1173-9.

3. Eddy DM. Investigational treatments. How strict should we be? JAMA 278:179-85.

4. Ellrodt G. Evidence-based disease management. JAMA 1978; 20:1687-92. 